\title{
A Facile and Efficient Tamarind Juice Catalyzed One-Pot Synthesis of Benzopyranopyrimidines in Aqueous Medium Just by Grinding: A Green Chemistry Approach
}

\author{
Y. I. SHAIKH ${ }^{1 *}$, S. S. SHAIKH ${ }^{2}$, KHURSHEED AHMED ${ }^{1}$, \\ G. M. NAZERUDDIN ${ }^{1}$ and V. S. SHAIKH ${ }^{3}$
}

${ }^{1}$ Department of Chemistry and P.G. Research Centre, Abeda Inamdar Sr. College, Pune 411002, Inda. 2Department of Chemistry, Gramonnati Mandal's Arts, Comm. \& Sci. College, Narayangaon 410504, India.

3Department of Chemistry, Nowrosjee Wadia College, Pune 411002, India.

${ }^{*}$ Corresponding author E-mail: sheray2k@gmail.com

http://dx.doi.org/10.13005/ojc/360308

(Received: March 29, 2020; Accepted: June 10, 2020)

\begin{abstract}
A comprehensive and efficient Tamarind (Tamarindous indica) juice catalysed one pot synthesis of benzopyranopyrimidines in aqueous medium through condensation of 4-Hydroxycoumarin $(10 \mathrm{mmol})$, various substituted benzaldehydes $(10 \mathrm{mmol})$ ) and urea/thiourea $(20 \mathrm{mmol})$ just by grinding at ambient condition is reported in excellent yield of the product. This is a green chemistry approach, which is need of the hour.
\end{abstract}

Keywords: Multi-component reaction, Tamarind Juice, Benzopyranopyrimidine, Grinding Technique (Green Chemistry).

\section{INTRODUCTION}

The industrial revolution ${ }^{1}$ took place between 1760 to 1840 and transition of hand power to machine took place and all the chemical reactions were accomplished by using steam power means coal was used and due to materialistic approach of various industries nobody was bothered about pollution problem and as a result of this ill effects were observed on health of human beings and pollution of this planet was increasing day by day. All the scientists of the world came forward and new term was coined as Green Chemistry². So under the umbrella of green chemistry to accomplish any chemical reaction an organic/medicinal chemist has to follow green chemistry principles. It means they have to prefer multicomponent reaction ${ }^{3}$ than multi step reaction so that the atom economy of the reaction could be nicely controlled. Similarly a biocatalyst ${ }^{4}$ and water ${ }^{5}$ as a solvent is preferable. A reaction always should consume less energy i.e. supported by grinding technique ${ }^{6}$, micro-wave ${ }^{7}$ or ultrasound irradiation ${ }^{8}$ with excellent yield of the product.

Pyrimidine derivatives have attracted interest of an organic/medicinal chemist because

This is an Open Access article licensed under a Creative Commons license: Attribution 4.0 International (CC- BY). Published by Oriental Scientific Publishing Company @ 2018 
of their various biologically active properties ${ }^{9,10,11}$. Patil et al., ${ }^{12,13}$ reported the synthesis of pyrimidine using lemon juice and pineapple juice as a catalyst. Whereas Nazeruddin et al., ${ }^{14,15}$ reported the same reaction catalyzed by Tamarind juice under ultra sound and Grape juice just by grinding at ambient conditions.

Coumarin derivatives possess a variety of biologically active properties ${ }^{16}$, and if these two rings i.e. pyrimidine and coumarin are condensed the resulting heterocyclic compound's derivatives would posses' enhanced biological activities. Various protocols are reported in the literature ${ }^{17}$. However, most of them do not follow the basic principles of green chemistry. In the present work rather it is continuation of our earlier work ${ }^{18}$ there is condensation of tautomeric form of 4-Hydroxycoumarin 1, aromatic aldehyde 2 and urea/thiourea $\mathbf{3}$ in aqueous medium catalyzed by Tamarind juice just by grinding technique furnishes benzopyrimidines 4 in excellent yields (Scheme 1).

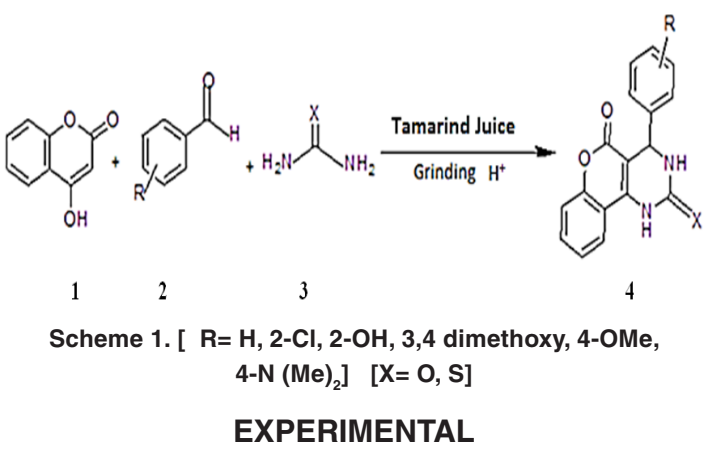

The chemicals required to carry out this research work were purchased from Merck and Loba and used as it is. Melting points were determined by an open capillary method and are uncorrected. IR spectra were recorded on Perkin-Elmer FT-IR1710 instrument. ${ }^{1} \mathrm{H}$ NMR spectra were recorded using $\mathrm{CDCl}_{3}$ or $\mathrm{DMSOd}_{6}$ as a solvent and TMSas an internal standard either on BrukerAC-200 MHz or Bruker MSL-300 MHz instrument. Elemental analyses were determined by an elemental.

\section{Preparation of aqueous extract of tamarind juice}

The tamarind fruit's pulp (without cover and seeds) was purchased from the local market and out of it $5 \mathrm{~g}$ of the pulp was soaked in $50 \mathrm{~mL}$ water for half an hour followed by pealing with hand to take out the extract and centrifuged by (REMI RM-12C).
The clear portion of the aqueous extract $(\mathrm{pH}=3)$ was used as catalyst.

\section{General procedure for the preparation of Benzopyranopyrimidines}

The aromatic aldehyde $(2 \mathrm{mmol})$ and 4-hydroxycoumarin ( $2 \mathrm{mmol}$ ) and urea/thiourea $(4 \mathrm{mmol})$ were taken in mortar followed by addition of $10 \mathrm{ml} 10 \%$ tamarind juice the mixture was ground for appropriate time (Table 1). The completion of the reaction was monitored by thin layer chromatography. The crude product was filtered, washed by water and dried under vacuum followed by crystallization using ethanol as a solvent.

\section{RESULTS AND DISCUSSION}

An environmentally benign procedure for synthesis of benzopyranopyridines is developed by condensing, 4-hydroxycoumarin, aromatic aldehydes and urea/thiourea in aqueous medium just by grinding using tamarind juice as a catalyst (Scheme 1).There is a complex role of tamarind juice in promoting the coupling reaction. Moreover, acidic nature of the juice $\left(\mathrm{H}^{+}\right.$ion) accelerates the reaction. The mechanism of the reaction is suggested in Scheme 2. Further, the methodology is general because, it accommodates various substituted benzaldehydes with electron donating and electron withdrawing groups (Table 1).Furthermore, the products are obtained in excellent yields just by filtration followed by crystallization.

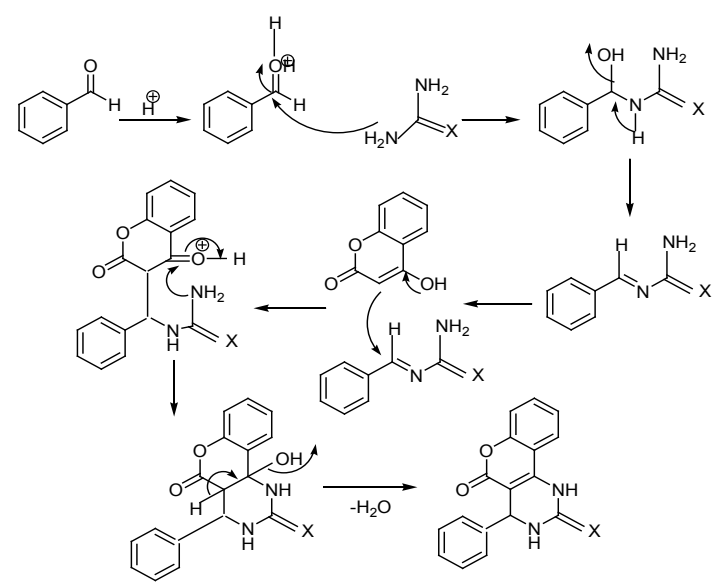

Scheme 2. Mechanism of the reaction, The reaction is initiated by protonation of the aldehyde followed by a formation of condensed product with urea/thiourea, which reacts with 4-hydroxy coumarin to accomplish the final product, the benzopyranopyridine 


\begin{tabular}{|c|c|c|c|c|c|c|}
\hline $\begin{array}{l}\text { Entry of the } \\
\text { product } 4 a-4 h\end{array}$ & $R$ & $x$ & $\begin{array}{l}\text { Time } \\
(\min )\end{array}$ & $\begin{array}{l}\text { Yield } \\
(\%)\end{array}$ & $\begin{array}{l}\text { Obs. } \\
\text { m.p. }{ }^{\circ} \mathrm{C}\end{array}$ & $\begin{array}{l}\text { Lit. } \\
\text { m.p. }{ }^{\circ} \mathrm{C}\end{array}$ \\
\hline $4 a$ & $\begin{array}{c}\text { phenyl } \\
\text { 2-chloro phenyl }\end{array}$ & $\mathrm{O}$ & 30 & 92 & $160-162$ & $162-164^{19}$ \\
\hline $4 b$ & 4-chloro phenyl & $\mathrm{O}$ & 20 & 85 & $205-207$ & $205-207^{18}$ \\
\hline $4 c$ & 3,4-dimethoxy phenyl & O & 20 & 95 & $198-200$ & $197-198^{20}$ \\
\hline $4 d$ & 2-hydro phenyl & $\mathrm{O}$ & 40 & 90 & $268-270$ & $270-272^{19}$ \\
\hline $4 e$ & 4-methoxy phenyl & S & 40 & 91 & $169-171$ & $169-171^{18}$ \\
\hline $4 f$ & 4-N,N dimethyl phenyl & S & 35 & 90 & $264-266$ & $264-266^{18}$ \\
\hline $4 g$ & & $\mathrm{O}$ & 45 & 90 & $239-241$ & $240-242^{20}$ \\
\hline $4 \mathrm{~h}$ & phenyl & $\mathrm{S}$ & 30 & 91 & $188-190$ & $188-189^{19}$ \\
\hline
\end{tabular}

Structures of the products were confirmed by comparing their M.P./B.P. and spectral data with authentic samples, which are as follows.

\section{Spectral and Elemental Analysis data}

3,4-Dihydro-4-phenyl-1H-chromeno [4, 3-d] pyrimidine-2, 5-dione (4a). White solid, m.p. 160-1620 C. IR: $(\mathrm{KBr})\left(v_{\max } \mathrm{cm}^{-1}\right)=3410,2924,2727$, 2360, 1654, 1459, 1379, 1303, 1154, 1075, 964, 722 $\mathrm{cm}^{-1} ;{ }^{1} \mathrm{H}-\mathrm{NMR}(\mathrm{DMSO}): \delta(\mathrm{ppm})=6.34(\mathrm{~s}, 1 \mathrm{H}, \mathrm{CH}), 7.16-$ $7.59(\mathrm{~m}, 9 \mathrm{H}, \mathrm{Ar}-\mathrm{H}), 7.87$ (brs, $1 \mathrm{H}, \mathrm{NH}), 7.9$ (brs, $1 \mathrm{H}$, $\mathrm{NH}$ ); Anal. Calcd for $\mathrm{C}_{17} \mathrm{H}_{12} \mathrm{~N}_{2} \mathrm{O}_{3}: \mathrm{C}, 69.86 ; \mathrm{H}, 4.14$; N, 9.58\%. Found: C, 69.92; H, 4.22; N, 9.70\%.

4-(2-Chlorophenyl)-3, 4-dihydro-1Hchromeno [4, 3-d] pyrimidine-2, 5-dione (4b). White solid, m.p. $205-207^{\circ} \mathrm{C}$. IR: $(\mathrm{KBr})\left(v_{\max } \mathrm{cm}^{-1}\right)=$ 3402, 3300, 3040, 1682, 1607, 1159, 1219, 1060, 757, 652, 553, 493, $453 \mathrm{~cm}^{-1}$; ${ }^{1} \mathrm{H}-\mathrm{NMR}$ (DMSO): $\delta(\mathrm{ppm})=6.14(\mathrm{~s}, 1 \mathrm{H}, \mathrm{CH}), 7.1-7.53(\mathrm{~m}, 8 \mathrm{H}, \mathrm{Ar}-\mathrm{H})$, 7.84(brs, $1 \mathrm{H}, \mathrm{NH}), 7.86$ (brs, $1 \mathrm{H}, \mathrm{NH}$ ); Anal. Calcd for $\mathrm{C}_{17} \mathrm{H}_{11} \mathrm{CIN}_{2} \mathrm{O}_{3}: 62.49 ; \mathrm{H}, 3.39 ; \mathrm{N}, 8.57 \%$. Found: C, $62.58 ; \mathrm{H}, 3.51 ; \mathrm{N}, 8.65 \%$.

4-(4-Chlorophenyl)-3, 4-dihydro-1Hchromeno [4, 3-d] pyrimidine-2, 5-dione (4c). White solid, m.p. $198-200^{\circ} \mathrm{C}$. IR: $(\mathrm{KBr})\left(v_{\max } \mathrm{cm}^{-1}\right)=3400$, 3079 , 2893, 2839, 2733, 2615, 1668, 1608, 1566, $1491,1450,1350,1309,1217,1093,1055,1014$, $765,671 \mathrm{~cm}^{-1} ;{ }^{1} \mathrm{H}-\mathrm{NMR}$ (DMSO): $\delta(\mathrm{ppm})=6.45(\mathrm{~s}, 1 \mathrm{H}$, $\mathrm{CH}), 7.28-7.66(\mathrm{~m}, 8 \mathrm{H}, \mathrm{Ar}-\mathrm{H}), 7.94(\mathrm{brs}, 1 \mathrm{H}, \mathrm{NH})$, 7.98(brs, $1 \mathrm{H}, \mathrm{NH}$ ); Anal. Calcd for $\mathrm{C}_{17} \mathrm{H}_{11} \mathrm{CIN}_{2} \mathrm{O}_{3}: \mathrm{C}$, 62.49; H, 3.39; N, 8.57\%. Found: C, 62.55; H, 3.47; $\mathrm{N}, 8.66 \%$.

3,4-Dihydro-4-(3,4-dimethoxyphenyl)-1Hchromeno[4,3-d]pyrimidine-2,5-dione (4d). White solid, m.p. 268-270 ${ }^{\circ} \mathrm{C}$. IR: $(\mathrm{KBr})\left(v_{\max } \mathrm{cm}^{-1}\right)=3415$, 2938, 2835, 2728, 2611, 2363, 1699, 1617, 1506, 1453, 1346, 1244, 1187, 1126, 1010, 907, 763, 506, $452 \mathrm{~cm}^{-1} ;{ }^{1} \mathrm{H}-\mathrm{NMR}$ (DMSO): $\delta(\mathrm{ppm})=3.54(\mathrm{~s}, 3 \mathrm{H}, \mathrm{OCH}$ 3), $3.69\left(\mathrm{~s}, 3 \mathrm{H}, \mathrm{OCH}_{3}\right), 6.25(\mathrm{~s}, 1 \mathrm{H}, \mathrm{CH}), 6.64-7.86(\mathrm{~m}, 7 \mathrm{H}$, Ar-H), 7.88 (brs, $1 \mathrm{H}, \mathrm{NH}), 7.89(\mathrm{brs}, 1 \mathrm{H}, \mathrm{NH})$; Anal. Calcd for $\mathrm{C}_{19} \mathrm{H}_{16} \mathrm{~N}_{2} \mathrm{O}_{5}: \mathrm{C}, 64.77 ; \mathrm{H}, 4.58 ; \mathrm{N}, 7.95 \%$. Found: C, 64.73, H, 4.69; N, 7.99\%.

1, 2, 3, 4-Tetrahydro-4-(2-hydroxyphenyl)2-thioxochromeno [4, 3-d] pyrimidin-5-one (4-e). Pale yellow, m.p. $169-171^{\circ} \mathrm{C}$. IR: $(\mathrm{KBr})\left(v_{\max } \mathrm{cm}^{-1}\right)$ $=3411,3071,2362,1752,1606,1488,1449$, 1389, 1343, 1241, 1271, 1039, 940, 865, 752, 465 $\mathrm{cm}^{-1}$; ${ }^{1} \mathrm{H}-\mathrm{NMR}$ (DMSO): $\delta(\mathrm{ppm})=3.3(\mathrm{brs}, 1 \mathrm{H}, \mathrm{OH})$, 6.89-7.85(m,9H,Ar-H), 8.32(brs, $1 \mathrm{H}, \mathrm{NH}$ ), 10.67 (brs, $1 \mathrm{H}, \mathrm{NH}$ ); Anal. Calcd for $\mathrm{C}_{17} \mathrm{H}_{12} \mathrm{~N}_{2} \mathrm{O}_{3} \mathrm{~S}: \mathrm{C}, 62.95$; $\mathrm{H}, 3.73 ; \mathrm{N}, 8.64 ; \mathrm{S}, 9.89 \%$. Found: C, 63.07; H, 3.84; N, 8.73; S, 9.91\%.

1,2, 3, 4-Tetrahydro-4-(4-methoxyphenyl)-2thioxochromeno [4, 3-d] pyrimidin-5-one (4-f). White solid, m.p. $264-266^{\circ} \mathrm{C}$. IR: $(\mathrm{KBr})\left(v_{\max } \mathrm{cm}^{-1}\right)=3401$, $3072,1967,1714,1626,1608,1583,1489,1450$, $1330,1344,1344,1242,1217,1174,1039,941$, 
866, 808, 754, 678, 624, $464 \mathrm{~cm}^{-1}$; ${ }^{1} \mathrm{H}-\mathrm{NMR}$ (DMSO): $\delta(\mathrm{ppm})=3.78\left(\mathrm{~s}, 3 \mathrm{H}, \mathrm{OCH}_{3}\right), 6.43(\mathrm{~s}, 1 \mathrm{H}, \mathrm{CH}), 6.86-$ 7.71(m,8H,Ar-H), 8.02(brs, 1H, NH), 8.06(brs,1H, NH); Anal. Calcd for $\mathrm{C}_{18} \mathrm{H}_{14} \mathrm{~N}_{2} \mathrm{O}_{3} \mathrm{~S}$ : C, 63.89; $\mathrm{H}, 4.17 ; \mathrm{N}, 8.28$; S, 9.48\%. Found:C, 63.96; H, 4.26, N, 8.38; S, 9.53\%.

4-(4-(Dimethylamino) phenyl)-3, 4-dihydro1H-chromeno [4, 3-d] pyrimidine-2, 5-dione (4-g). Pinkish brown, m.p.239-241 ${ }^{\circ} \mathrm{C}$. IR: $(\mathrm{KBr})\left(v_{\max } \mathrm{cm}^{-1}\right)=$ 3426, 3088, 2885, 2727, 2609, 1662, 1610, 1568, 1523, 1450, 1348, 1307, 1207, 1184, 1089, 1053, 958, 906, 765, $744 \mathrm{~cm}^{-1} ;{ }^{1} \mathrm{H}-\mathrm{NMR}$ (DMSO): $\delta(\mathrm{ppm})=3$ $.19\left(\mathrm{~s}, 6 \mathrm{H}, \mathrm{CH}_{3}\right), 6.44(\mathrm{~s}, 1 \mathrm{H}, \mathrm{CH}), 7.24-7.37(\mathrm{~m}, 8 \mathrm{H}, \mathrm{Ar}-\mathrm{H})$, 7.86(brs, $1 \mathrm{H}, \mathrm{NH}), 7.90(\mathrm{brs}, 1 \mathrm{H}, \mathrm{NH})$; Anal. Calcd for $\mathrm{C}_{19} \mathrm{H}_{17} \mathrm{~N}_{3} \mathrm{O}_{3}$ : C, 68.05; $\mathrm{H}, 5.11 ; \mathrm{N}, 12.53 \%$. Found: $\mathrm{C}$, 68.13; H, 5.18; N, 12.60\%.

1,2,3, 4-Tetrahydro-4-phenyl-2-thioxo chromeno [4, 3-d] pyrimidin-5-one (4-h). White solid, m.p.188-190 C. IR: $(\mathrm{KBr})\left(v_{\max } \mathrm{cm}^{-1}\right)=3450,2923$, 2854, 1656, 1463, 1377, 1303, 1155, 970, $727 \mathrm{~cm}^{-1}$; ${ }^{1} \mathrm{H}-\mathrm{NMR}$ (DMSO): $\delta(\mathrm{ppm})=6.36(\mathrm{~s}, 1 \mathrm{H}, \mathrm{CH}), 7.17-7.60$ (m, 9H,Ar-H), 7.88(brs,1H,NH), 7.91 (brs, 1H, NH); Anal. Calcd for $\mathrm{C}_{17} \mathrm{H}_{12} \mathrm{~N}_{2} \mathrm{O}_{2} \mathrm{~S}: \mathrm{C}, 66.22 ; \mathrm{H}, 3.92 ; \mathrm{N}$, 9.08;S, $10.40 \%$. Found: C, 66.31; H, 3.95; N, 9.13; S, $10.44 \%$.

\section{CONCLUSION}

A facile, efficient, green chemistry protocol for the synthesis of benzopyranopyrimidines is developed with simple procedure, low cost, high yield of the product and above all such protocols are the need of the hour.

\section{ACKNOWLEDGEMENT}

Authors are thankful Maharashtra Cosmopolitan Education Society Pune for the financial support.

\section{Conflict of interest}

The authors have declared that there is no conflict of interest.

\section{REFERENCES}

1. Anthony WrigleyE; Journal of Interdisciplinary History., 2018, 49(01), 09-42.

2. Anastas, P.T.; Warner,J.C.;Oxford University Press, Oxford., 1998.

3. Shaikh, S.R.;Nazeruddin, G.M.; J.Chemical and Pharmaceutical Research., 2014, 6 (12), 505-534.

4. $\quad$ Andrade, L. H.; Utsunomiya, S.; Omori, A. T.; Porto, A. L. M.; Comasseto, J. V. J.; Mol. Catal. B: Enzyme., 2006, 38, 84-90.

5. Sherman, J.; Chin, B.; Huibers, P. D. T.; GarciaValls, R.; Hatton, T. A.; 1998, 106, 253-271.

6. Bose, A.K.;Pednekar, S.;Ganguly, S.N., Chakraborty, G.;Manhas, M.S.; Tetrahedron Lett., 2004, 45, 8351-8353.

7. Nazeruddin, G. M.;Mulani, S.S.;Pandharpatte, M. S.; Chinese journal of Chemical Society., 2012, 59, 645-649.

8. Al-Kadasi, A. M. A.;Nazeruddin, G.M; Asian Journal of Chemistry., 2011, 23(7), 3155-3157.

9. Motos, L.H.S.; Masson, F.T.; Simeoni, L.A.;Homem-de-Mello,M.; Eur J Med Chem. 2018, 143, 1779-1789.

10. Kappe, C.O.; Tetrahedron., 1993, 49(32),
6937-6963.

11. Kappe, C. O.; Acc. Chem. Res., 2000, 33, 879-888.

12. Patil,S.; Jadhav, S.D.; and Deshmukh, M.B.; Archive of Applied Science., 2011, 3, 203-208.

13. Patil, S.; Jadha, S.D.; and Mane, S.Y.; Int. J. of Org. Chemistry., 2011, 1, 125-131.

14. Nazeruddin,G.M.;Shaikh,Y.I.; Der Pharmacia Sinica., 2014, 5(6), 64-68.

15. Nazeruddin,G.M.; Mulani,S.S.; Shaikh, Y.I.; Shaikh, S.S.K.;Int. J of Scientific Research in Science and Technology., 2017, S.I.3,46-50.

16. Gupta, A.S.;Prabhu, B.S.;Phull, M.S.;Indian J. Chem. Sect. B., 1996, 35, 170-171.

17. Matache, M.;Dobrota, C.;Bogdan, N. D.; Dumitru, I.;Ruta, L.L.; Paraschivescu, C. C.; Farcasanu, I. C.;Baciu,I.; Funeriu, D. P.; Tetrahedron., 2009, 65, 5949-5957.

18. Al-Kadasi,A.; and Nazeruddin,G. M.; J. of Chem. Pharma. Res., 2013, 5(7), 204-210.

19. Brahmbhatt, D.I.; Roliji, G.B.; Pandya, S.U.; Pandya, U.R.; Indian J. Chem., 1999, 38, 839.

20. Kidwai, M.;Sapra,P.; Synthetic communications., 2002, 32, 1639-1645. 